\title{
A density functional theory study of the relative stability of the iron disulfide polymorphs, Pyrite and Marcasite
}

\author{
D Spagnoli, ${ }^{1}$ K Refson, ${ }^{2}$ K Wright, ${ }^{1}$ and J.D Gale ${ }^{1}$ \\ ${ }^{1}$ Nanochemistry Research Institute, Department of Chemistry, \\ Curtin University of Technology, PO Box U1987, Perth, WA 6845, Australia \\ ${ }^{2}$ STFC Rutherford Appleton Laboratory, Didcot, Oxfordshire OX11 0QX, UK
}

Submitted: November 2009

\begin{abstract}
The performance of density functional theory (DFT) has been widely examined with regard to its ability to predict the properties of minerals, though less attention has been given to the correct determination of the relative stability of structurally similar polymorphs. Here a detailed examination is performed of the numerical and theoretical factors that may influence the structure and relative energetics of two such polymorphs of iron disulfide, namely pyrite and marcasite, within density functional theory. Both the local density approximation and commonly used generalized gradient approximation exchange-correlation functionals, such as PBE, are found to predict that marcasite is more stable than pyrite, at variance with experiment. Allowing for the zero point energy of vibration fails to remedy this discrepancy. While inclusion of a
\end{abstract}


sufficiently large Hubbard $U$ parameter for iron is found to reverse the stability, this comes at the expense of a very poor description of other properties. Examination of three generalized gradient approximations developed specifically for the solid-state, namely AM05, Wu-Cohen and PBEsol, demonstrates that all of these functionals offer a superior description of the structures and relative energies of pyrite and marcasite through correctly predicting that the former is the ground state phase at ambient conditions.

PACS number: 71.15.Mb, 91.60.-x, 61.50.-f, 63.20.dk, 71.10.Fd, 71.15.Dx, 


\section{INTRODUCTION}

Iron sulfide minerals are common in nature and can exist in a variety of forms with varying stoichiometries that ranges from the sulfur deficient mackinawite $\left(\mathrm{FeS}_{1-\mathrm{x}}\right)$ through iron deficient pyrrhotites $\left(\mathrm{Fe}_{1-\mathrm{x}} \mathrm{S}\right)$ to pyrite $\left(\mathrm{FeS}_{2}\right)$. In the lower temperature environments of the Earth's surface the iron sulfide group is particularly complex and a number of metastable phases

become significant in biogeochemical processes. ${ }^{1}$ Iron sulfides provide evidence for processes and fluxes in the global biogeochemical sulfur cycle.

Iron disulfide occurs naturally as two polymorphs. The cubic pyrite form (FIG. 1a) (space group Pa3) is by far the most abundant of the iron disulfides, with the octahedrally coordinated Fe atoms at the corners and face centers of the cubic unit cell. ${ }^{2}$ The less abundant polymorph is the orthorhombic marcasite form (FIG. 1b) (space group Pnnm). The coordination environments of Fe and S atoms in marcasite are very similar to those in pyrite, while the difference between the two structures is found in the linking of Fe-centered octahedra. In the marcasite structure, the octahedra share edges forming chains parallel to the shortest of the three distinct crystallographic axes. Adjacent chains share vertices and the channels comprised by four adjacent chains are reduced to a rhomb-like cross-section by the bridging S-S bonds. ${ }^{3}$ The pyrite structure can be obtained from that of the marcasite by rotation of half the $S_{2}$ groups through $90^{\circ}{ }^{2}$ 
Commensurate with the above subtle structural distinction, the difference in the free energy of formation between the two polymorphs is very low, with pyrite being $0.04 \mathrm{eV} / \mathrm{FeS}_{2}$ more stable than marcasite at $298.15 \mathrm{~K} .{ }^{4}$ Pyrite can be synthesized both by high-temperature reaction between elemental $\mathrm{Fe}$ and $\mathrm{S}$, as well as by precipitation from aqueous solution. Marcasite can be synthesized at lower temperatures and more acidic conditions, leading to the general assumption that the formation and persistence of marcasite is not due to its thermodynamic stability but to kinetic factors. ${ }^{5-7}$ It is proposed by Tossell et al. that the effect of the $\mathrm{H}^{+}$ion is to withdraw two electrons from the $\mathrm{Fe}$ atom and thereby impart a distorted geometry to the three $\mathrm{Fe}$ atoms coordinated to each $\mathrm{S}$ atom of the disulfide group, ${ }^{8}$ thus promoting the formation of the orthorhombic marcasite structure.

Pyrite forms in a range of terrestrial environments including those associated with massive sulfide ore bodies. It can contain a range of impurities, including the so-called “invisible" gold that makes it economically important. ${ }^{9,}{ }^{10}$ In addition, pyrite is a semiconductor that can be $n$ - or $p$-type depending on the impurity species present. Pyrite is a material of considerable technological interest because of its application in photovoltaic devices. ${ }^{11,12}$ Phase purity is important in photovoltaic devices, since the unusually low open circuit voltage that has be observed for nanoparticulate pyrite has been attributed to the inclusion of the marcasite structure. ${ }^{13}$ Given the desire to ultimately probe the structural and phase variations of 
nanoparticles of iron disulfide, it is valuable to be able to perform accurate computer simulations

as a complement to experimental information. However, the thermodynamic and structural similarities of the two polymorphs of iron disulfide render this system to be a challenging one.

Although there have been several previous theoretical examinations of pyrite and/or marcasite, ${ }^{14}$ most studies avoid a discussion of the relative phase stability, indicating that this may be a problematic quantity. In this study we will critically examine the ability of density functional theory based calculations to describe the structure, properties, and especially the relative phase stabilities of pyrite and marcasite in order to provide guidance for reliable studies of such materials.

\section{METHODOLOGY}

In the present study, we report a first-principles investigation of the two polymorphs of iron disulfide, pyrite and marcasite, based on Kohn-Sham density functional theory (DFT). DFT has become the de facto standard for electronic structure theory in the solid-state, and has been widely utilized throughout physics, chemistry and biology. ${ }^{15}$ It has also been employed very successfully in the past to accurately describe the bulk, surface and electronic properties of numerous minerals. ${ }^{16-18}$ Pyrite itself has received much attention from computational methods, 
including DFT ${ }^{19-21}$ and interatomic potential based studies. ${ }^{22}$ In contrast, theoretical studies involving the marcasite phase are very limited, with one study comparing the thermodynamics of arsenic incorporation into pyrite and marcasite. ${ }^{23}$ The majority of DFT studies to date have only focused on one polymorph of this mineral, thereby avoiding the difficult issue of the relative phase stability of two very similar structures.

Recently, there has been a great deal of research into developing new exchangecorrelation functionals specifically designed for solid-state systems. To date, most studies in the field of minerals have utilized either the local density approximation to the exchange-correlation (XC) functional, or more recently gradient-corrected forms, such as the generalized gradient approximation (GGA) ${ }^{24}$. Although hybrid functionals are extensively used for molecular systems, the greater computational cost and, until recently, the more limited availability of Hartree-Fock exchange for periodic systems, has led to fewer studies of solids based on this methodology. ${ }^{25,}{ }^{26}$ As a result, the most commonly used XC functional type remains the generalized gradient approximation (GGA), such as the PBE functional. ${ }^{27,28}$

It is widely recognized that the majority of GGAs suffer from systematic errors for solids;

for example the lattice parameters are often overestimated by approximately $1 \% \cdot{ }^{27}$ This has led to a number of investigations into developing new XC functionals that are specifically designed for solids while staying within the GGA framework for reasons of computational expediency. 
Armiento and Mattsson presented an improved XC functional (AM05), which was derived from the energies of an Airy gas and the surface of jellium. ${ }^{24}$ This functional has been demonstrated to yield lattice parameters and bulk moduli that are superior to PBE and competitive with hybrid functionals. ${ }^{29} \mathrm{Wu}$ and Cohen explored the exchange part of the functional ${ }^{30,31}$ and modified the PBE form to improve the properties in the slowly varying density limit. This functional has also been shown to be more accurate than PBE for the lattice constants of solids. ${ }^{32}$ Most recently, Perdew et al. developed the PBEsol functional that retains the same functional form as the PBE functional. However, two parameters were modified in order to satisfy the gradient expansion for exchange that is more relevant for solids. ${ }^{27,28}$

In this study we explore a range of DFT-based methods, including the PBE, PBEsol, AM05 and Wu-Cohen XC functionals, in order to examine their description of the relative stabilities of pyrite and marcasite. The DFT computations were conducted using several different approaches in order to examine the influence of numerical approximations, such as pseudopotential construction and basis set. Given the similarity of the two phases, it is essential to ensure that numerical factors are not influencing the quality of the results. Reference calculations were performed first using the FP-LAPW methodology, within the program Elk, ${ }^{33}$ in order to validate the construction of the pseudopotentials for more extensive calculations based on this approximation in combination with a plane-wave basis set. Here the programs 
CASTEP ${ }^{34}$ and PWSCF ${ }^{35}$ were utilized. Finally, in order to examine the prospects for being able to perform reliable large-scale calculations within linear-scaling density functional theory, the SIESTA ${ }^{36}$ methodology and program was employed to perform calculations within the linear-combination of atomic orbitals approximation. The following four sub-sections give an overview of the methodology and the relevant convergence tests that were performed for each numerical implementation of DFT.

\section{A. FP-LAPW calculations}

All-electron full-potential linearized augmented-plane wave (FP-LAPW) calculations have been performed using the Elk code. ${ }^{33}$ The FP-LAPW method is among the most precise DFT-based methods for performing electronic structure calculations for crystal structures. ${ }^{37}$ The FP-LAPW method divides space into an interstitial region and non-overlapping muffin-tin (MT) spheres centered at the atomic sites. In the interstitial region, the basis set is described by planewaves, whereas in the MT spheres, the basis set is described by radial solutions of the one particle Schrödinger equation and their energy derivatives multiplied by spherical harmonics. A plane-wave cut-off in the interstitial region is limited by $k_{\max }=7.0 / R_{\min }$ (where $R_{\min }$ is the smallest muffin-tin radius in the unit cell), which corresponds to a value of $173 \mathrm{eV}$. The $k_{\max }$ 
value was increased, therefore increasing the cut-off from $173 \mathrm{eV}$ to $295 \mathrm{eV}$, to test for convergence. Inside the MT spheres, states are expanded up to the maximum angular quantum number $l_{\max }=12$. The muffin-tin radii were taken as 2.09 a.u. for iron and 1.96 a.u. for sulfur. Brillouin-zone integrations have been performed using Monkhorst-Pack grids of 8x8x8 and 8x6x4 for pyrite and marcasite, respectively.

The convergence tolerance for the forces of ions during structural optimization was set to $5 \times 10^{-5}$ and convergence of total energy was achieved when an absolute change was lower than $1 \times 10^{-4}$ Hartree. Presently analytic stresses are not available within Elk and so the unit cell parameters were optimized by sequential line searches, where the internal coordinates were fully relaxed for each cell configuration. For marcasite, where there are three independent cell parameters, the values were iteratively refined until all values were converged to three decimal places.

\section{B. Pseudopotential-plane-wave calculations}

Two different pseudopotential plane-wave implementations have been utilized in the

present work, namely CASTEP ${ }^{34}$ and PWSCF; ${ }^{35}$ both employ a plane-wave basis set for the expansion of the Kohn-Sham valence orbitals, while the combination of the core states and 
nuclei are represented by ultra-soft pseudopotentials. Considering first the specifics of the calculations performed using CASTEP, here the ultrasoft pseudopotentials are generated using an "on-the-fly" pseudopotential generator. This provides the advantage of being able to test the effect of changing the XC functional or the Hamiltonian self-consistently within the present calculations. The valance configuration considered for sulfur during pseudopotential generation was $3 s^{2} 3 p^{4}$, with a single ultrasoft s- and p-projector and a double ultrasoft d-projector. The atomic valence configuration for the iron atoms was $3 s^{2} 3 \mathrm{p}^{6} 3 \mathrm{~d}^{6} 4 \mathrm{~s}^{2}$, and thus includes the semicore states explicitly. The radii for the local and non-local components were both set to 1.9 a.u.

The kinetic energy cut-off was tested for convergence by increasing the energy from 300$1000 \mathrm{eV}$ in $25 \mathrm{eV}$ increments and convergence of the relative energies was achieved at $525 \mathrm{eV}$. The sampling of the Brillouin zone was carefully tested. The pyrite phase was tested with $4 \times 4 \times 4$, 8x8x8, 12x12x12 and 16x16x16 Monkhorst-Pack grids, ${ }^{38}$ whereas the marcasite phase was tested with Monkhorst-Pack grids of 8x6x4, 10x8x6 and 12x10x8. Based on these convergence tests, a kinetic energy cut-off of $525 \mathrm{eV}$ was used for both phases of iron disulfide with a 4x4x4 k-point grid for pyrite and an 8x6x4 k-point grid for the marcasite phase. The charge density cutoff was set to $2100 \mathrm{eV}$. The above convergence tests described were performed using the PBE XC functional. However, similar convergence properties were found for all XC functionals used. During geometry optimization, the structure was considered to be converged when the maximum 
forces on the atoms were lower than $0.02 \mathrm{eV} / \AA \AA$ and the difference in energy per atom was lower than $2 \times 10^{-5} \mathrm{eV}$.

For the calculations with PWSCF, previously constructed pseudopotentials for iron and sulfur were utilized (available from http://www.pwscf.org/pseudo.htm). The iron pseudopotential was generated for a partial ionized configuration of $3 d^{6.5} 4 s^{1} 4 p^{0}$, while the sulfur pseudopotential was generated for the same neutral configuration as reported for CASTEP. These pseudopotentials were generated based on the PBE functional, and therefore only used for calculations based on this GGA form. A series of convergence tests were performed to ensure that the kinetic energy cut-off and number of k-points were sufficiently high enough so that any increase would not affect the accuracy of the calculations. The kinetic energy cut-off was examined over the range of 524-1069 eV. Convergence of relative energy of pyrite and marcasite was achieved for a cut-off of $664 \mathrm{eV}$. The charge density cut-off was set to $2656 \mathrm{eV}$. Similar convergence tests of the Brillouin-zone sampling to those described above were repeated for the present numerical implementation, leading to the same choice of Monkhorst-Pack grids, as expected. 


\section{Linear-combination of atomic orbital calculations}

Here we examine the performance of the SIESTA ${ }^{36}$ methodology, which utilizes a linear combination of atomic orbitals (LCAO) to expand the Kohn-Sham wave functions. Nuclei and core electrons are replaced by norm-conserving pseudopotentials, which were generated according to the modified scheme of Troullier and Martins. ${ }^{39}$ The valence electronic configurations used in the pseudopotential generation were $3 s^{2} 3 p^{3.5} 3 d^{0.5}$ for sulfur and $3 p^{6} 4 s^{2} 3 d^{6}$ for iron. All pseudopotentials were regenerated for each XC functional used in order to ensure proper consistency.

The basis functions were obtained by finding the numerical pseudo-atomic orbitals (PAOs) of the isolated atoms enclosed within a soft-confined spherical potential. ${ }^{40}$ The basis sets employed were of double- $\zeta$ quality for iron and sulfur, except for the 3s and 3p orbitals of the sulfur and $3 d$ orbital of the iron where triple- $\zeta$ quality was required. The basis sets were optimized with respect to the pyrite structure with parameters of 0.005 Ry for the energy shift of radial confinement and a split-norm value of 0.1 . A full description of the basis sets used in this study is given in the Supplementary Material. ${ }^{41}$ An auxiliary real space mesh was used to 
evaluate terms involving the charge density. An equivalent plane-wave cut-off of 400 Ry was

used to represent the density.

\section{Results and Discussion}

\section{A. Structure and phase stability of pyrite and marcasite based on the PBE functional}

The structures of both pyrite and marcasite have been fully optimized with respect to the

lattice parameters and internal coordinates using the above numerical implementations of density

functional theory. As described in the section above, the relevant convergence criteria were rigorously tested before the geometry optimization calculations were performed. Using the GGA PBE exchange correlation function the lattice parameters of both phases of iron disulfide can be compared with experimental data (Table 1). The calculated lattice parameter for the cubic pyrite structure falls into two groups. The values derived using FP-LAPW and SIESTA are very similar to each other, while those obtained from the pseudopotential plane-wave approach are consistent, but indicate a lower unit cell volume. This difference may reflect the influence of the pseudopotential approximation, with the norm-conversing form better reproducing the full 
potential than the ultrasoft technique. Alternatively, the incompleteness of the atomic-orbital basis set may lead to a fortuitous cancellation of errors within the SIESTA case.

The fact that the PBE-based calculations either underestimate, or are close to, the experimental value might be considered unexpected, given that PBE typically overestimates lattice parameters by $1 \%$. However, the experimental value quoted is for a room temperature structure determination, while the theoretical data corresponds to absolute zero. The lattice parameters of pyrite will undergo expansion when subjected to higher temperatures. Experimental studies of the lattice parameters of pyrite at different temperatures can be found, however, the majority of temperatures reported are between 294 and $300 \mathrm{~K} .{ }^{42-44}$ If we take these lattice parameters as a function of temperature and extrapolate to zero Kelvin, we can estimate that the lattice parameter of pyrite in the low temperature limit is $5.281 \AA$. Thermal expansion of lattice parameters would not be completely linear, due to vibrational quantization, and so we can anticipate that the above value is a lower bound to the lattice parameter at absolute zero. Furthermore, natural samples of pyrite are often found to contain impurities ${ }^{45}$ and may be nonstoichiometric, ${ }^{46}$ and this will also effect the experimental lattice parameters. For marcasite, the lattice parameters are more consistent amongst the numerical implementations, with the values for CASTEP being in particularly good agreement with those from the FP-LAPW method. Again 
the calculated values are relatively close to the experimental ones due to the lack of the thermal expansion correction.

The values of lattice parameters obtained in the present work are comparable with other values found in the literature. Blanchard et al. used the CASTEP and SIESTA codes to calculate the lattice parameters of pyrite using the PBE exchange-correlation functional and calculated lattice parameters of 5.406 and $5.449 \AA$, respectively, ${ }^{47}$ which is consistent with our data for the plane-wave result. The lower value for the SIESTA methodology in the present work is due to the use of improved numerical factors. Recently Blanchard et al. calculated the lattice parameters of pyrite using the PWSCF code and the same XC functional to obtain a value of $5.407 \AA .{ }^{48}$

Regardless of the variations in lattice parameter, it is clear that the PBE functional predicts that the most favorable phase of iron disulfide is marcasite (Table 1), which is in disagreement with experiment. To investigate the origin of this disagreement with regard to the phase stability, several possible refinements to the theoretical methodology have been explored, as described in the following sections.

\section{B. Vibrational contribution to the energy of iron disulfide phases}


The previous calculations considered the relative phase stability between pyrite and marcasite based only on the $0 \mathrm{~K}$ static energy. To investigate whether finite temperature and zero point energy effects might influence the relative stability, we have computed the phonons of both pyrite and marcasite. Here we use two methodologies to calculate the dynamical matrix, namely linear-response, as implemented in the PWSCF code, and central finite differencing of the analytic first derivatives within the SIESTA and CASTEP methodologies. The phonons were calculated at the $\Gamma$-point of the Brillouin zone and from the sum of the vibrational frequencies the zero point energy (ZPE) of the crystal lattice can be calculated:

$$
Z P E=\sum \omega\left(\frac{\hbar}{2}\right)
$$

where $\omega$ is the vibrational frequency and $\hbar$ is Planck's constant. A discussion of the influence of phonon dispersion will be presented later. The number and symmetries of the Raman and infrared-active modes of pyrite have been determined using group theoretical methods. ${ }^{49}$ The irreducible representations of the vibrations of pyrite are:

$$
\Gamma=A_{g}+E_{g}+3 T_{g}+2 A_{u}+2 E_{u}+6 T_{u}
$$


The gerade vibrations $\left(A_{g}+E_{g}+3 T_{g}\right)$ are all active in the Raman spectrum, and the ungerade vibrations are divided into the IR-active $\left(5 T_{u}\right)$ and optically inactive $\left(2 A_{u}+2 E_{u}\right)$ modes, plus the three acoustic modes. Group theoretical examination of the marcasite structure yields six Ramanallowed and seven IR-active zone center phonon modes: ${ }^{50}$

$$
\Gamma=2 A_{g}+2 B_{1 g}+B_{2 g}+B_{3 g}\left(+2 A_{u}+B_{1 u}+3 B_{2 u}+3 B_{3 u}\right)
$$

The seven IR-active modes consist of the irreducible representations $\left(B_{1 u}+3 B_{2 u}+3 B_{3 u}\right)$ and have had their symmetry coordinates determined by Lutz and Willich. ${ }^{51}$

The harmonic phonon frequencies of pyrite and marcasite, computed at the $\Gamma$-point of the Brillouin zone, are shown in Tables 2 and 3 respectively. There is good agreement between the frequencies computed by both implementations of DFT used, as well as with experimental data and previous DFT studies of the pyrite phase. ${ }^{48,52-54}$ The majority of the phonon frequencies for pyrite are lower than the corresponding experimental values, while for marcasite there is a more variable distribution.

From equation (1) we can calculate the ZPE for both phases of iron disulfide (Table 4). It is readily apparent that the zero point energy of pyrite is greater than that of marcasite, regardless 
of method, and thus this term will further contribute to the discrepancy with experiment. Of course, the above values are computed based on the $\Gamma$-point alone, and so it is important to consider the influence of phonon dispersion. The highest frequency modes for both pyrite and marcasite correspond to the stretching modes of the $\mathrm{S}_{2}$ dimers and thus these will dominate the zero point energy. Because these modes are highly localized to a molecular sub-unit, they only exhibit weak dispersion across the Brillouin zone and therefore sampling across reciprocal space will not significantly change this result.

These results have indicated conventional DFT methodology using the PBE functional gives reasonably good agreement between the experimental and calculated lattice parameters, as well as for the phonon frequencies of the two phases of iron disulfide. However, the relative stability between pyrite and marcasite is not correctly predicted and inclusion of the vibrational contribution to the thermodynamics fails to correct this error.

\section{Effect of the Hubbard $U$ on the relative phase stability of pyrite and marcasite}

Treatment of iron within density functional theory has long been recognized as problematic, in part because of the strong correlation/self-interaction error for the localized d- 
electrons. ${ }^{55}$ Consequently, this factor needs to be investigated to determine whether it is responsible for the failure to predict the correct order of phase stability.

The DFT $+U$ method, developed in the 1990 s, $^{56,57}$ is a well-established model to describe the electronic structure of strongly correlated systems. The Hubbard $U$ parameter is the Coulomb energy cost to place two electrons at the same site, which alters the one-electron potential locally for the specified orbitals, here those of the Fe $3 d$ shell, thus reducing the hybridization with the S ligands. The DFT $+U$ method has been successfully applied to describing an improved band gap, magnetic moment and density of states, as compared with experimental data, for $\mathrm{Fe}_{2} \mathrm{O}_{3}$ ( $\alpha$-hematite), ${ }^{55}$ and improved band gaps for the rutile and anatase phases of $\mathrm{TiO}_{2}{ }^{58}$

Here we have performed calculations for the GGA+ $U$ method within the PWSCF code. ${ }^{35}$ $U$ values from 0 to $8 \mathrm{eV}$ have been examined, spanning the typical range for this quantity, and the effect on lattice parameters and relative phase stability explored. Details of the calculations are identical to those performed previously, except for the inclusion of the $U$ contribution.

Although the $U$ parameter can be determined based on a linear-response approach, ${ }^{59}$ this leads to a structure dependent value and thus potentially different values for pyrite and marcasite. This strategy, which leads to distinct Hamiltonians for different solids, is not appropriate for the 
calculation of relative energies of phases. Hence, in the present work we explore the influence of a common $U$ value within the range of typically utilized values.

As the Hubbard $U$ parameter is increased from 0 to $8 \mathrm{eV}$ the lattice parameters and cell volume increases for both pyrite and marcasite (FIG 2). As seen in other studies involving iron sulfides, ${ }^{60}$ the introduction of the Hubbard $U$ parameter, has significant effects on the lattice parameter. To our knowledge there is one other study that utilizes the Hubbard $U$ parameter to study the bulk properties of pyrite. ${ }^{61}$ Persson et al. used a Hubbard $U$ value of $3 \mathrm{eV}$ and found that the cell volume also increased compared to the conventional DFT and obtained a closer value to experimental data. Our simulations indicate that the best agreement with experimental lattice parameters is found with a $U$ value of $2 \mathrm{eV}$ for pyrite.

The relative phase stability between pyrite and marcasite is found to stay in favor of marcasite until the $U$ value reaches $6.5 \mathrm{eV}$ (Table 5). However, at this $U$ value the unit cell volume is $8.5 \%$ larger than the experimental values for both phases of iron disulfide, and therefore this is unlikely a represent a physically realistic choice of the Hubbard parameter.

\section{Influence of the exchange-correlation functional}


From the previous section, it is interesting to note that the change in the volume of the cell does influence the relative stability of the phases, even for such similar polymorphs. The unit cell volume of the two phases is an important factor to consider and as previously mentioned the PBE functional typically leads to an overestimation of the lattice cell parameters. Recent developments in DFT calculations of solids have included the introduction of exchangecorrelation functionals for solids and these new functionals lead to a contraction of lattice parameters for certain solids, ${ }^{62}$ compared with the PBE XC functional. Typically these functionals yield results closer to those obtained from LDA, which itself often describes the density well, but is inaccurate for other properties. To investigate the influence of the functional, we have examined the performance of three relatively new GGAs that are specifically designed with the solid-state or surfaces in mind; specifically these are, in chronological order, AM05, the Wu-Cohen (WC), and PBEsol functionals.

The PBEsol functional was implemented into the CASTEP and SIESTA codes for the present work, while AM05 has also been added to SIESTA using the routines made available by the original authors. ${ }^{24}$ The most recent version of the all-electron Elk code also includes all three functionals to be examined. As an initial test, we compare the results of geometry optimizations for pyrite and marcasite based only on PBEsol, and return to consideration of all three functionals subsequently. 
The lattice parameters resulting from the use of the PBEsol XC functional all contract in length (Table 6) compared with the equivalent calculations based on the use of the PBE XC functional. The magnitude of this contraction is quite significant, representing approximately a 2\% decrease in lattice parameters on average, between the PBE and PBEsol XC functionals. The resulting decrease in cell volume coincides with a correct prediction of the order of phase stability between the two polymorphs. All three numerical implementations concur that pyrite is now the most stable phase, which implies that the unit cell volume of two very similar polymorphs may be of critical importance. This is further demonstrated by the fact that the ultrasoft plane-wave calculations yield significantly smaller lattice parameters than the FP-LAPW and norm-conserving pseudopotential/LCAO methods, leading to a magnification of the energy difference by an order of magnitude.

As all three numerical implementations of DFT result in qualitatively the same behavior, we have used the SIESTA methodology to examine whether the same correct prediction of the thermodynamics is also observed with the AM05 and Wu-Cohen XC functionals (Table 7). All three XC functionals give good agreement between the lattice parameters, and both the PBEsol and AM05 XC functionals correctly predict pyrite as being more stable than marcasite. However, the Wu-Cohen functional still predicts that marcasite is the ground state, though the magnitude of the energy difference is close to the level of the numerical uncertainty, let alone the likely 
accuracy of density functional theory. To demonstrate the numerical sensitivity, the CASTEP code also has the Wu-Cohen functional implemented and a geometry optimization calculation of the two phases yields an energy difference of $0.0065 \mathrm{eV}^{-} \mathrm{FeS}_{2}$ in favor of pyrite.

There are many studies in the literature that have calculated the lattice parameters and bulk moduli of a variety of solids. ${ }^{32,63,64}$ It is well known that the local-density approximation (LDA) also underestimates the lattice parameters of solids by $1 \% .{ }^{27}$ Hence, if the unit cell volume is the only factor in relative phase stability then it might be expected that the LDA would yield the correct ordering for pyrite and marcasite. To test this we have performed geometry optimization calculations of both phases of iron disulphide using the LDA XC functional in SIESTA and obtained a lattice parameter of $5.400 \AA$ for pyrite and lattice parameters of 3.389, 4.444 and $5.407 \AA$ for marcasite. The cell volume contracts compared with the experimental data and the PBE XC functional, as expected. However, the relative phase stability still favors marcasite by $0.031 \mathrm{eV} / \mathrm{FeS}_{2}$. Therefore, it is not only the contraction in volume of the unit cell, but the form of these new functionals with respect to the limiting behavior in different regions of the reduced density gradient, which accounts for the correct prediction of the phase stability between pyrite and marcasite. Each functional is formed in a different way and a full and comprehensive review of the construction and performance of the new GGA functionals designed for solids can be found elsewhere in the literature. ${ }^{32,62,63}$ 
From this study we observe that all the GGA XC functionals designed for solids predict the correct phase stability ordering of pyrite and marcasite. Regardless of numerical issues, the important observation is the trend towards favoring the stability of pyrite, and the strong correlation of the energy difference with the predicted volume, though with this not being the exclusive factor, as demonstrated by the failure of the local density approximation.

\section{Conclusions}

We have used a variety of DFT-based methodologies and functionals to study the structure and relative phase stability of the two polymorphs of iron disulfide, pyrite and marcasite. The use of the PBE XC functional, and almost certainly most other GGAs of the same generation, leads to the increase of unit cell volume of both phases, which in turn results in an incorrect prediction of relative phase stability. Consideration of the phonon contributions to the free energy is found not to be important in regard to the relative phase stability. Furthermore, the addition of a Hubbard $U$ fails to correct the stability difference for physically plausible values, though it does alter the unit cell volume.

The use of any of the new generation of GGA functionals, such as PBEsol, AM05 or WuCohen, results in a contraction of lattice parameters and increase in stability of pyrite with 
respect to marcasite bringing the DFT results in line with experimental data. Moreover, the AM05 XC functional has been shown to be very promising for molecular systems, such as water.

65 This indicates that this functional can be potentially used for a wide range of materials, including condensed phases, with the possibility of improved relative energetics spanning a variety of different applications.

From this initial study we propose that XC functionals derived specifically for solids or surfaces should always be used when determining the relative phase stabilities of structures, and is especially important for very similar polymorphs. However, a more comprehensive study should be conducted with other minerals, such as the anatase and rutile phases of titanium dioxide, to determine whether this observation is more generally true, and which of the XC functionals best describes the structure and thermodynamics of very similar polymorphs.

\section{Acknowledgements}

The authors would like to thank the ARC for funding under the Discovery Program, as well as NCI and iVEC for provision of computational resources. 


\section{REFERENCES}

D. Rickard and G. W. Luther, Chem. Rev. 107, 514 (2007).

A. R. Lennie and D. J. Vaughan, American Mineralogist 77, 1166 (1992).

E. Makovicky, in Sulfide Mineralolgy and Geochemistry (Mineralogical Soc America, Chantilly, 2006), Vol. 61, p. 7.

M. W. Chase, C. A. Davies, J. R. Downey, D. J. Frurip, R. A. McDonald, and A. N. Syverud, Journal of Physical and Chemical Reference Data 14, 1 (1985).

M. A. A. Schoonen and H. L. Barnes, Geochimica et Cosmochimica Acta 55, 1495 (1991).

M. A. A. Schoonen and H. L. Barnes, Geochimica et Cosmochimica Acta 55, 1505 (1991).

M. A. A. Schoonen and H. L. Barnes, Geochimica et Cosmochimica Acta 55, 3491 (1991).

J. A. Tossell, D. J. Vaughan, and J. K. Burdett, Physics and Chemistry of Minerals 7, 177 (1981).

L. M. Maddox, G. M. Bancroft, M. J. Scaini, and J. W. Lorimer, American Mineralogist 83, 1240 (1998).

D. Paktunc, D. Kingston, A. Pratt, and J. McMullen, Canadian Mineralogist 44, 213 (2006).

A. Ennaoui, S. Fiechter, C. Pettenkofer, N. Alonsovante, K. Buker, M. Bronold, C. Hopfner, and H. Tributsch, Solar Energy Materials and Solar Cells 29, 289 (1993).

C. Wadia, Y. Wu, S. Gul, S. K. Volkman, J. Guo, and A. P. Alivisatos, Chemistry of Materials 21, 2568 (2009).

G. W. Luther, Geochimica Et Cosmochimica Acta 55, 2839 (1991).

D. W. Bullett, Journal of Physics C-Solid State Physics 15, 6163 (1982).

M. J. Gillan, Contemp Phys 38, 115 (1997).

K. Refson, P. R. Tulip, and S. J. Clark, Phys. Rev. B 73, 12 (2006).

M. Nolan, J. E. Fearon, and G. W. Watson, Solid State Ionics 177, 3069 (2006).

P. J. D. Lindan, N. M. Harrison, M. J. Gillan, and J. A. White, Phys. Rev. B 55, 15919 (1997).

M. Blanchard, M. Alfredsson, J. Brodholt, K. Wright, and C. R. A. Catlow, Geochimica Et Cosmochimica Acta 71, 624 (2007).

J. Muscat, A. Hung, S. Russo, and I. Yarovsky, Phys. Rev. B 65, 054107 (2002).

I. Opahle, K. Koepernik, and H. Eschrig, Computational Materials Science 17, 206 (2000).

N. H. de Leeuw, S. C. Parker, H. M. Sithole, and P. E. Ngoepe, Journal of Physical Chemistry B 104, 7969 (2000).

M. Reich and U. Becker, Chem. Geol. 225, 278 (2006).

R. Armiento and A. E. Mattsson, Phys. Rev. B 72, 085108 (2005).

J. Paier, M. Marsman, K. Hummer, G. Kresse, I. C. Gerber, and J. G. Angyan, Journal of Chemical Physics 124, 13 (2006). 
M. J. Gillan, D. Alfe, S. De Gironcoli, and F. R. Manby, J. Comput. Chem. 29, 2098 (2008).

J. P. Perdew, A. Ruzsinszky, G. I. Csonka, O. A. Vydrov, G. E. Scuseria, L. A. Constantin, X. L. Zhou, and K. Burke, Physical Review Letters 100, 136406 (2008).

J. P. Perdew, A. Ruzsinszky, G. I. Csonka, O. A. Vydrov, G. E. Scuseria, L. A. Constantin, X. L. Zhou, and K. Burke, Physical Review Letters 102, 039902 (2009).

A. E. Mattsson, R. Armiento, J. Paier, G. Kresse, J. M. Wills, and T. R. Mattsson, Journal of Chemical Physics 128, 084714 (2008).

Z. G. Wu and R. E. Cohen, Phys. Rev. B 73, 235116 (2006).

Z. G. Wu and R. E. Cohen, Phys. Rev. B 78, 197102 (2008).

P. Haas, F. Tran, and P. Blaha, Phys. Rev. B 79, 085104 (2009).

K. Dewhurst, et al., http://elk.sourceforge.net

S. J. Clark, M. D. Segall, C. J. Pickard, P. J. Hasnip, M. J. Probert, K. Refson, and M. C. Payne, Zeitschrift Fur Kristallographie 220, 567 (2005).

S. Baroni, et al., http://www.pwscf.org/.

J. M. Soler, E. Artacho, J. D. Gale, A. Garcia, J. Junquera, P. Ordejon, and D. Sanchez-Portal, J PhysCondens Mat 14, 2745 (2002).

F. Drief, A. Tadjer, D. Mesri, and H. Aourag, Catalysis Today 89, 343 (2004).

H. J. Monkhorst and J. D. Pack, Phys. Rev. B 13, 5188 (1976).

N. Troullier and J. L. Martins, Phys. Rev. B 43, 1993 (1991).

J. Junquera, O. Paz, D. Sanchez-Portal, and E. Artacho, Phys. Rev. B 64, 235111 (2001).

See EPAPS Document No. [] for the basis sets used in the simulations of the SIESTA code. For more information on EPAPS see http://www.aip.org/pubservs/epaps.html

E. D. Stevens, M. L. Delucia, and P. Coppens, Inorganic Chemistry 19, 813 (1980).

M. Birkholz, S. Fiechter, A. Hartmann, and H. Tributsch, Phys. Rev. B 43, 11926 (1991).

W. Paszkowicz and J. A. Leiro, Journal of Alloys and Compounds 401, 289 (2005).

P. K. Abraitis, R. A. D. Pattrick, and D. J. Vaughan, Int. J. Miner. Process. 74, 41 (2004).

K. Ellmer and C. Hopfner, Philos. Mag. A-Phys. Condens. Matter Struct. Defect Mech. Prop. 75, 1129 (1997).

M. Blanchard, K. Wright, J. D. Gale, and C. R. A. Catlow, J. Phys. Chem. C 111, 11390 (2007).

M. Blanchard, F. Poitrasson, M. Méheut, M. Lazzeri, F. Mauri, and E. Balan, Geochimica et Cosmochimica Acta 73, 6565 (2009).

H. D. Lutz and P. Willich, Zeitschrift Fur Anorganische Und Allgemeine Chemie 405, 176 (1974).

H. D. Lutz and B. Muller, Physics and Chemistry of Minerals 18, 265 (1991).

H. D. Lutz and P. Willich, Zeitschrift Fur Naturforschung Section a-a Journal of Physical Sciences 30, 1458 (1975). 
M. Blanchard, M. Alfredsson, J. Brodholt, G. Price, K. Wright, and C. Catlow, J Phys Chem B 109, 22067 (2005).

W. Buhrer, E. Lafougere, and H. Lutz, Journal of Physics and Chemistry of Solids 54, 1557 (1993).

C. Sourisseau, R. Cavagnat, and M. Fouassier, J Phys Chem Solids 52, 537 (1991).

G. Rollmann, A. Rohrbach, P. Entel, and J. Hafner, Phys. Rev. B 69, 12 (2004).

V. I. Anisimov, J. Zaanen, and O. K. Andersen, Phys. Rev. B 44, 943 (1991).

A. I. Liechtenstein, V. I. Anisimov, and J. Zaanen, Phys. Rev. B 52, R5467 (1995).

N. A. Deskins and M. Dupuis, Phys. Rev. B 75, 10 (2007).

M. Cococcioni and S. de Gironcoli, Phys. Rev. B 71, 035105 (2005).

A. J. Devey, R. Grau-Crespo, and N. H. de Leeuw, Phys. Rev. B 79, 7 (2009).

K. Persson, G. Ceder, and D. Morgan, Phys. Rev. B 73, 115201 (2006).

G. I. Csonka, J. P. Perdew, A. Ruzsinszky, P. H. T. Philipsen, S. Lebegue, J. Paier, O. A. Vydrov, and J. G. Angyan, Phys. Rev. B 79, 155107 (2009).

P. Haas, F. Tran, P. Blaha, K. Schwarz, and R. Laskowski, Phys. Rev. B 80, 195109 (2009).

F. Tran, R. Laskowski, P. Blaha, and K. Schwarz, Phys. Rev. B 75, 115131 (2007).

A. E. Mattsson and T. R. Mattsson, Journal of Chemical Theory and Computation 5, 887 (2009). 


\section{FIGURES}
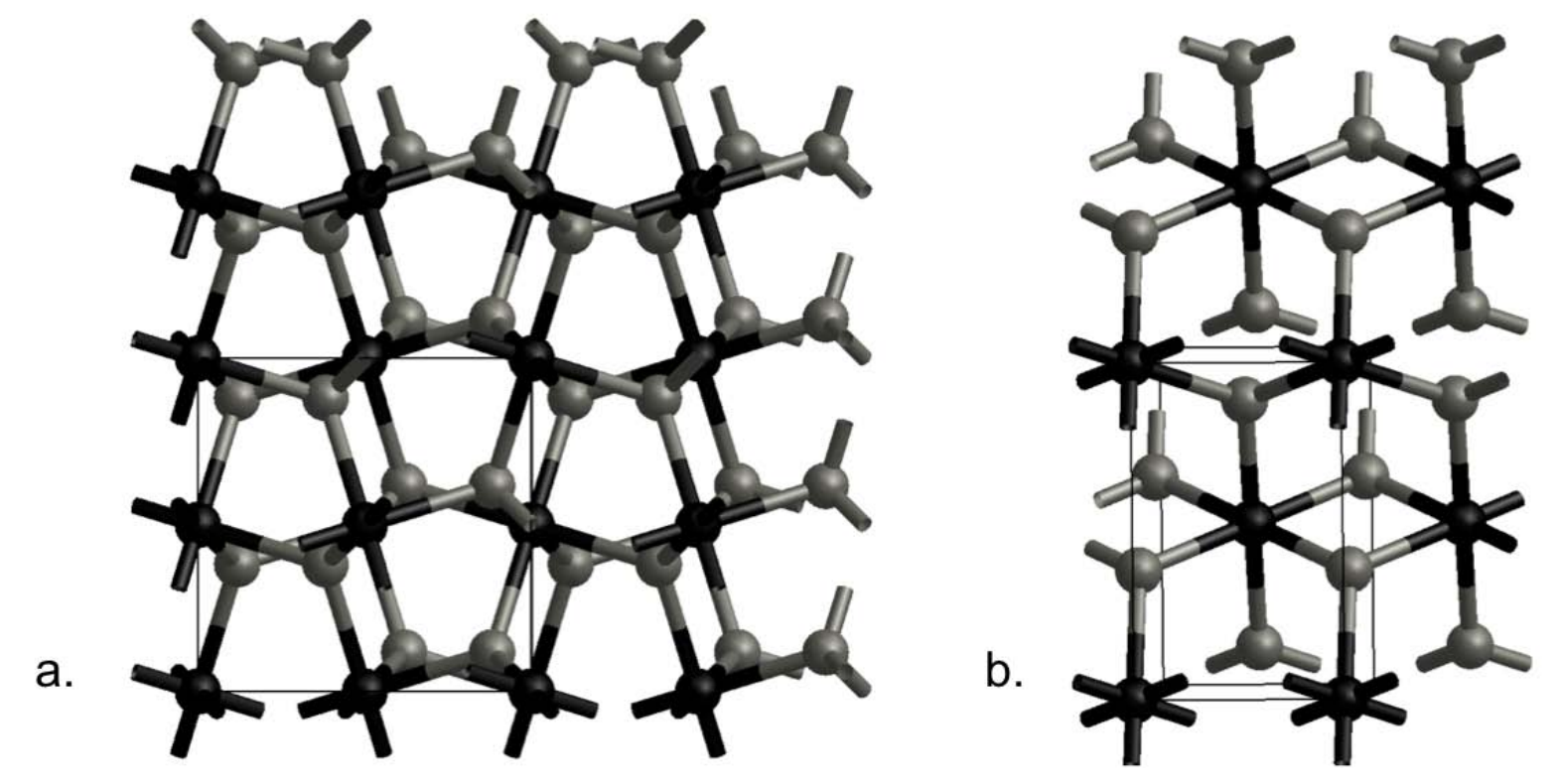

FIG 1: Bulk structure of a) pyrite (unit cell volume-159.04 $\AA^{3}$ ) and b) marcasite (unit cell volume-80.65

$\AA^{3}$ ) with the unit cell of each mineral defined by the black lines. Black spheres are iron atoms and grey spheres are sulfur atoms 

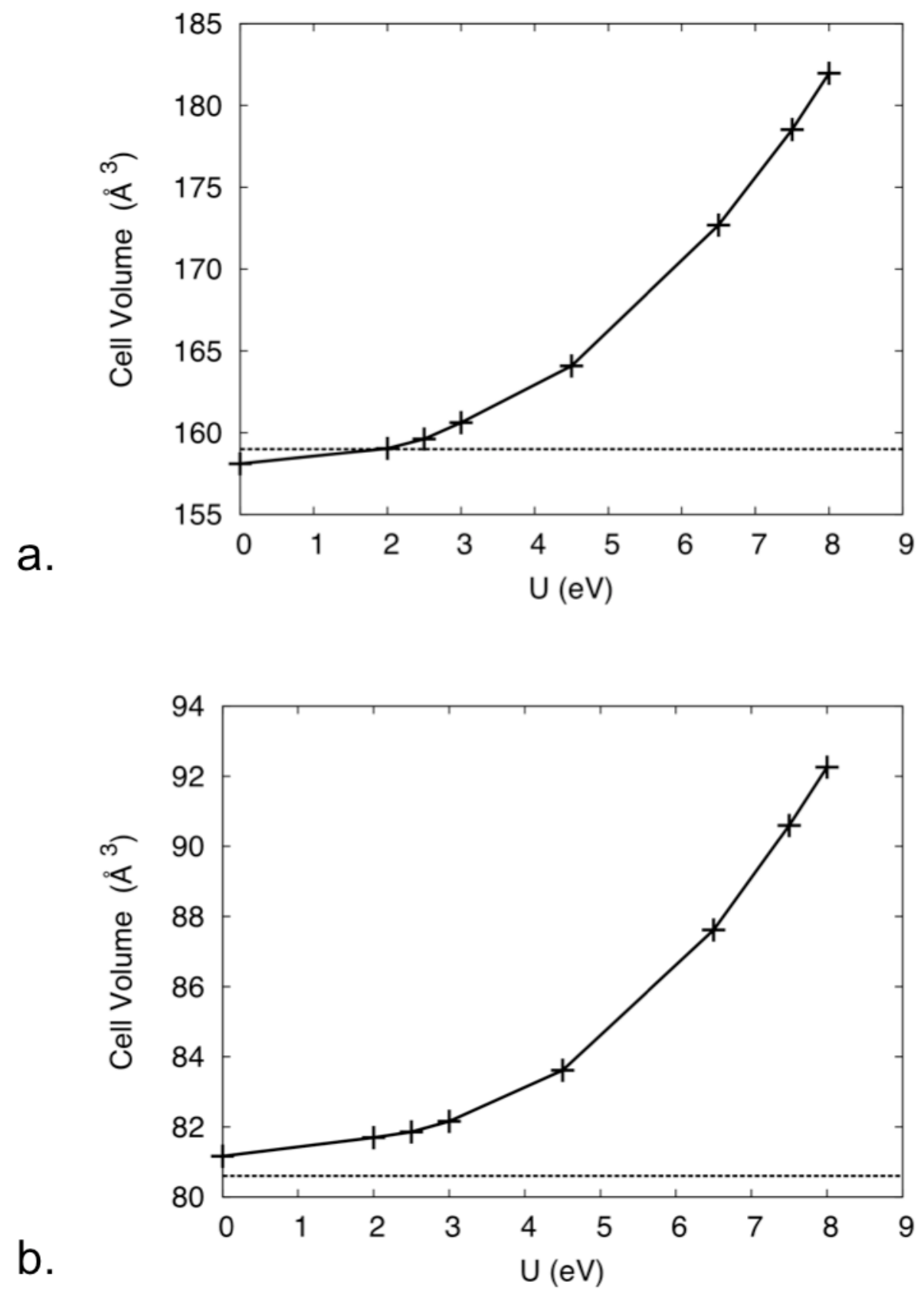

FIG 2: Calculated cell volume for a) pyrite and b) marcasite as a function of Hubbard parameter U. The dashed lines represent the experimental unit cell volume. 


\section{TABLES}

\begin{tabular}{|c|c|c|c|c|c|}
\hline & Pyrite & \multicolumn{3}{|c|}{ Marcasite } & \\
\hline & \multirow{2}{*}{ Lattice Parameter } & \multicolumn{3}{|c|}{ Lattice Parameter } & \multirow{2}{*}{$\begin{array}{l}\text { Relative energy difference } \\
\qquad{\mathrm{eV} / \mathrm{FeS}_{2}}^{*}\end{array}$} \\
\hline & & a $(\AA)$ & $\mathrm{b}(\AA)$ & c $(\AA)$ & \\
\hline Experimental $^{3}$ & 5.418 & 3.386 & 4.443 & 5.424 & - \\
\hline CASTEP & 5.403 & 3.390 & 4.435 & 5.407 & 0.026 \\
\hline PWSCF & 5.407 & 3.387 & 4.434 & 5.404 & 0.033 \\
\hline SIESTA & 5.414 & 3.398 & 4.453 & 5.420 & 0.037 \\
\hline Elk & 5.417 & 3.386 & 4.449 & 5.432 & 0.028 \\
\hline
\end{tabular}

TABLE 1: Lattice parameters for the optimized unit cell of the two phases of iron disulfide, as computed according to different numerical implementations of density functional theory with the PBE XC functional. Relative energies for the phases are also given where this represents the difference between the final relaxed lattice energies of pyrite and marcasite; a positive value indicates that marcasite is more stable than pyrite. 


\begin{tabular}{|c|c|c|c|c|c|c|}
\hline Mode & Active Mode & Symmetry & PWSCF & CASTEP & SIESTA & Experimental ${ }^{53}$ \\
\hline 4 & & $\mathrm{~A}_{\mathrm{u}}$ & 203 & 213 & 209 & 199 \\
\hline $5-7$ & IR & $\mathrm{T}_{\mathrm{u}}$ & 215 & 216 & 212 & 215 \\
\hline $8-9$ & & $\mathrm{E}_{\mathrm{u}}$ & 236 & 238 & 239 & 225 \\
\hline $10-12$ & IR & $\mathrm{T}_{\mathrm{u}}$ & 283 & 287 & 281 & 289 \\
\hline 13 & & $\mathrm{~A}_{\mathrm{u}}$ & 323 & 318 & 323 & 310 \\
\hline $14-15$ & Raman & $E_{g}$ & 328 & 327 & 327 & 344 \\
\hline $16-18$ & IR & $\mathrm{T}_{\mathrm{u}}$ & 331 & 330 & 333 & 343 \\
\hline $19-21$ & Raman & $\mathrm{T}_{\mathrm{g}}$ & 341 & 341 & 334 & 344 \\
\hline $22-23$ & & $\mathrm{E}_{\mathrm{u}}$ & 355 & 360 & 354 & 396 \\
\hline 24 & Raman & $A_{g}$ & 359 & 364 & 363 & 382 \\
\hline $25-27$ & IR & $\mathrm{T}_{\mathrm{u}}$ & 368 & 365 & 363 & 402 \\
\hline $28-30$ & Raman & $\mathrm{T}_{\mathrm{g}}$ & 378 & 382 & 374 & 387 \\
\hline $31-33$ & IR & $\mathrm{T}_{\mathrm{u}}$ & 387 & 388 & 381 & 407 \\
\hline 34-36 & Raman & $\mathrm{T}_{\mathrm{g}}$ & 427 & 446 & 419 & 433 \\
\hline
\end{tabular}


TABLE 2: Calculated and experimental frequencies of the transverse optical modes of pyrite $\left(\mathrm{cm}^{-1}\right)$ using the PBE XC functional

\begin{tabular}{|c|c|c|c|c|c|c|}
\hline Mode & Active Mode & Symmetry & PWSCF & CASTEP & SIESTA & Experimental $^{54}$ \\
\hline 4 & & $\mathrm{~A}_{\mathrm{u}}$ & 207 & 210 & 211 & in. \\
\hline 5 & IR & $\mathrm{B}_{2 \mathrm{u}}$ & 248 & 257 & 256 & 325 \\
\hline 6 & IR & $\mathrm{B}_{3 \mathrm{u}}$ & 279 & 279 & 297 & 293 \\
\hline 7 & Raman & $A_{g}$ & 317 & 282 & 297 & 323 \\
\hline 8 & IR & $\mathrm{B}_{2 \mathrm{u}}$ & 323 & 314 & 317 & 404 \\
\hline 9 & Raman & $\mathrm{B}_{3 \mathrm{~g}}$ & 339 & 336 & 318 & 367 \\
\hline 10 & Raman & $\mathrm{B}_{2 \mathrm{~g}}$ & 342 & 338 & 320 & 308 \\
\hline 11 & IR & $\mathrm{B}_{3 \mathrm{u}}$ & 360 & 340 & 361 & 353 \\
\hline 12 & IR & $\mathrm{B}_{3 \mathrm{u}}$ & 373 & 355 & 364 & 387 \\
\hline 13 & Raman & $\mathrm{B}_{1 \mathrm{~g}}$ & 377 & 377 & 370 & - \\
\hline 14 & Raman & $\mathrm{B}_{1 \mathrm{~g}}$ & 382 & 384 & 374 & 377 \\
\hline 15 & IR & $\mathrm{B}_{2 \mathrm{u}}$ & 385 & 394 & 377 & 432 \\
\hline 16 & Raman & $A_{g}$ & 388 & 397 & 396 & 386 \\
\hline 17 & IR & $\mathrm{B}_{1 \mathrm{u}}$ & 409 & 406 & 398 & 404 \\
\hline
\end{tabular}




\begin{tabular}{|l|l|l|l|l|l|l|}
\hline 18 & Raman & $\mathrm{B}_{1 \mathrm{~g}}$ & 455 & 455 & 455 & - \\
\hline
\end{tabular}

TABLE 3: Calculated and experimental frequencies of the transverse optical modes of marcasite $\left(\mathrm{cm}^{-1}\right)$ using the PBE XC functional (in. = inactive vibration)

\begin{tabular}{|c|c|c|}
\hline Code & ZPE Pyrite $\mathrm{eV} / \mathrm{FeS}_{2}$ & ZPE Marcasite $\mathrm{eV} / \mathrm{FeS}_{2}$ \\
\hline PWSCF & 0.169 & 0.161 \\
\hline CASTEP & 0.171 & 0.159 \\
\hline SIESTA & 0.168 & 0.158 \\
\hline
\end{tabular}

TABLE 4: Calculated zero point energies from $\Gamma$-point phonon calculations for pyrite and marcasite using the PBE XC functional 


\begin{tabular}{|c|c|}
\hline $\mathrm{U}(\mathrm{eV})$ & ${\text { Relative energy difference } \mathrm{eV} / \mathrm{FeS}_{2}{ }^{*}}^{*}$ \\
\hline 0 & 0.031 \\
\hline 2.0 & 0.030 \\
\hline 2.5 & 0.028 \\
\hline 3.0 & 0.024 \\
\hline 4.5 & 0.012 \\
\hline 6.5 & -0.010 \\
\hline 7.5 & -0.021 \\
\hline 8.0 & -0.027 \\
\hline
\end{tabular}

TABLE 5: Relative stability between the pyrite and marcasite phases of iron disulfide as a function of

Hubbard U parameter. ${ }^{*}$ The relative energy is the difference between the final relaxed lattice energy of pyrite less that of marcasite; therefore, a positive value indicates marcasite as being more stable than pyrite. 


\begin{tabular}{|c|c|c|c|c|c|}
\hline & Pyrite & \multicolumn{3}{|c|}{ Marcasite } & \\
\hline & & \multicolumn{3}{|c|}{ Lattice Parameter } & \multirow{2}{*}{$\begin{array}{l}\text { Relative energy difference } \\
\qquad{\mathrm{eV} / \mathrm{FeS}_{2}{ }^{*}}^{*}\end{array}$} \\
\hline & a $(\AA)$ & a $(\AA)$ & $\mathrm{b}(\AA)$ & с $(\AA)$ & \\
\hline CASTEP & 5.315 & 3.340 & 4.355 & 5.324 & -0.0117 \\
\hline SIESTA & 5.331 & 3.344 & 4.385 & 5.347 & -0.0008 \\
\hline Elk & 5.333 & 3.342 & 4.387 & 5.384 & -0.0014 \\
\hline
\end{tabular}

TABLE 6: Lattice parameters of the relaxed unit cell for the two phases of iron disulfide, as well as the relative phase stability between pyrite and marcasite, as calculated using the PBEsol XC functional. ${ }^{*}$ The relative energy is quoted as the difference between the final relaxed lattice energy of pyrite less that of marcasite; a negative value indicates that pyrite is more stable than marcasite. 


\begin{tabular}{|c|c|c|c|c|c|}
\hline \multirow{2}{*}{ SIESTA } & Pyrite & \multicolumn{3}{|c|}{ Marcasite } & \multirow{2}{*}{ Relative energy difference } \\
\cline { 2 - 5 } XC functional & Lattice Parameter & \multicolumn{3}{|c|}{ Lattice Parameter } \\
\cline { 2 - 5 } & $\mathrm{a}(\AA)$ & $\mathrm{eV} / \mathrm{FeS}_{2}{ }^{*}$ \\
\hline PBEsol & 5.331 & 3.344 & 4.385 & 5.347 & \multirow{2}{*}{$\mathrm{c}(\AA)$} \\
\hline AM05 & 5.318 & 3.345 & 4.367 & 5.333 & -0.0008 \\
\hline Wu-Cohen & 5.345 & 3.351 & 4.397 & 5.358 & 0.0038 \\
\hline
\end{tabular}

TABLE 7: Lattice parameters of the relaxed unit cell for the two phases of iron disulfide, with corresponding exchange-correlation functional and the relative phase stability between pyrite and marcasite. ${ }^{*}$ The relative energy is the difference between the final relaxed lattice energy of pyrite less that of marcasite; a positive value indicates marcasite as being more stable than pyrite. 